\title{
Notfallmedizin up2date
}

$2 \cdot 2017$

\section{Allgemeine und organisatorische Aspekte 1}

\section{Neubewertung extraglottischer Atemwegshilfsmittel in der Notfallmedizin}

Arnd Timmermann Sebastian G. Russo 


\section{Unter dieser Rubrik sind bereits erschienen:}

Außerklinische Beatmung - Herausforderungen für den Rettungsdienst A. Lechleuthner, E. Singer, A. Geißler Heft 1/2017

Heilverfahren der gesetzlichen Unfallversicherung A. Hogan, S. Kuhnen, M. Münzberg, P. A. Grützner Heft 1/2017

Präklinische Versorgung akuter Blutungen nach schwerem Trauma B. Hußmann, U. Fochtmann, S. Lendemans Heft 4/2016

Hygiene im Rettungsdienst K. Strerath, B. Christiansen Heft 2/2016

Update: Beatmung im Rettungsdienst M. Strunden, T. Wieser, E. Nufer, T. Kerner Heft 2/2016

Erweiterte Maßnahmen für Erwachsene (Adult Advanced Life Support) J.-T. Gräsner, B. Bein Heft 1/2016

Basismaßnahmen bei Erwachsenen und Anwendung automatischer externer Defibrillatoren A. Bohn, S. Seewald, J. Wnent Heft 1/2016

Postreanimationsphase nach der neuen Leitlinie A. Schneider, B. Böttiger Heft 1/2016

Ambulante Behandlung im Rettungsdienst T. Beckmeier, M. Neupert, A. Bohn Heft 4/2015

Schnittstelle Notaufnahme: Optimierungen an der Nahtstelle Präklinik/Klinik F. Hilbig, A. Gries, T. Hartwig, M. Bernhard Heft 3/2015

Prähospitale Analgesie beim Erwachsenen B. Hossfeld, S. Holsträter, M. Bernhard, L. Lampl, M. Helm, M. Kulla Heft 3/2015

Handlungsempfehlung zur prähospitalen Notfallnarkose beim Erwachsenen, M. Bernhard, B. Bein, B. Böttiger, A. Bohn, M. Fischer, J. Gräsner, J. Hinkelbein, C. Kill, C. Lott, E. Popp, M. Roessler, A. Schaumberg, V. Wenzel, B. Hossfeld Heft 2/2015

Management des schwierigen Atemwegs unter Extrembedingungen E. Cavus, C. Byhahn, D. Meininger, V. Dörges Heft 2/2015

\section{ALLES ONLINE LESEN}

Mit der eRef lesen Sie Ihre Zeitschrift: online wie offline, am PC und mobil, alle bereits erschienenen Artikel. Für Abonnenten kostenlos! https://eref.thieme.de/notfall-u2d
TEMS - Taktische Medizin im Rahmen von Einsätzen der Strafverfolgungsbehörden B. Hossfeld, F. Josse, R. Bohnen, A. Garling, L. Lampl, M. Helm Heft 1/2015

Psychische Belastungen und Bewältigungsstrategien in der präklinischen Notfallversorgung H. Karutz, V. Blank-Gorki Heft 4/2014

Abbruch einer Reanimation im Rettungsdienst K. Kaerlein, A. Bohn, R. Lukas Heft 4/2014

Der Leitende Notarzt - etablierte Konzepte und neue Anforderungen H. Marung, T. Birkholz, M. Dittmar Heft 4/2014

Gebäudeeinsturz. Vernetzter Einsatz zur Rettung Verschütteter - Teil 2 D. Marten, M. Markus, D. Gümbel, M. Reinhardt, S. Weiss, K. Sieber, B. Domres Heft 3/2014

Leitsymptom akute Atemnot - präklinischer Fokus C. Möller, F. Sayk Heft 3/2014

Postreanimationsbehandlung M. Fischer, H. Roth, J.-C. Schewe, S. Rauch Heft 2/2014

Gebäudeeinsturz. Vernetzter Einsatz zur Rettung Verschütteter - Teil 1 D. Marten, M. Markus, D. Gümbel, M. Reinhardt, S. Weiss, K. Sieber, B. Domres Heft 2/2014

Laienreanimationsunterricht an Schulen A. Bohn, G. Rücker, R.-P. Lukas, H. Van Aken, J. Breckwoldt Heft 1/2014

Schweigen ist gefährlich H. Marung Heft 1/2014

Präklinische Versorgung und Management von Explosionsverletzungen T. Holsträter, S. Holsträter, D. Rein, M. Helm, B. Hossfeld Heft 3/2013

Der arztbegleitete Interhospitaltransport F. Reifferscheid Heft 2/2013

MANV - Strategien bei Großschadensereignissen D. Gümbel, C. G. Wölfl, J. Beneker, A. Ekkernkamp, G. Matthes Heft 2/2013

Mehr Überlebende durch mehr Wissen J.-T. Gräsner, J. Wnent, S. Seewald, A. Bohn, J. Scholz, B. Böttiger, M. Fischer Heft 2/2013

\section{JETZT FREISCHALTEN}

Sie haben Ihre Zeitschrift noch nicht freigeschaltet? Ein Klick genügt: www.thieme.de/eref-registrierung 


\section{Neubewertung extraglottischer Atemwegshilfsmittel in der Notfallmedizin}

Arnd Timmermann, Sebastian G. Russo

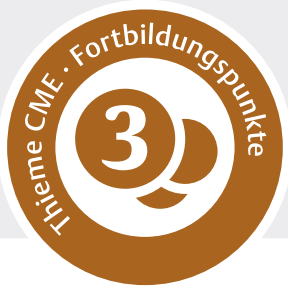

Dieser Artikel gibt einen Überblick über die klinischen Grundlagen von extraglottischen Atemwegshilfen (EGA), beschreibt den potenziell sinnvollen Einsatz in der präklinischen Notfallmedizin, stellt die derzeit aktuellen Studien vor, analysiert mögliche Schwachstellen hinsichtlich der Art der verwendeten Atemwegshilfen und beleuchtet die Ausbildung der medizinischen Notfallteams. Auch der rettungsdienstliche Einsatz von Atemwegshilfen bei Kindern wird dargestellt.

\begin{tabular}{|ll}
\hline \multicolumn{2}{|l}{ ABKÜRZUNGEN } \\
CPR & kardiopulmonale Reanimation \\
DGAI & $\begin{array}{l}\text { Deutsche Gesellschaft für Anästhesio- } \\
\text { logie und Intensivmedizin e. V. }\end{array}$ \\
& extraglottische Atemwegshilfe \\
EGA & endotracheale Intubation \\
ETI & Endotrachealtubus \\
ETT & hypopharyngealer Verschlussdruck \\
HPL & Atemwegshilfe vom Typ Larynxmaske \\
LMA & Larynxtubus \\
LT & Gesichtsmaskenbeatmung \\
MB & Notarzteinsatzfahrzeug \\
NEF & oropharyngealer Leckagedruck \\
OLP & Rettungstransportwagen \\
RTW & supraglottische Atemwegshilfe \\
SGA & \\
&
\end{tabular}

\section{Einleitung}

„Das Atemwegsmanagement ist eine der Kernkompetenzen der Notfallmedizin, da ohne offene Atemwege und einen adäquaten Gasaustausch alle weiteren Maßnahmen vergeblich werden".

So richtig dieser Satz von Burkhard Dirks [1] ist, beschreibt er leider auch ein Dilemma der Notfallmedizin: Die Sicherung der Atemwege ist präklinisch im Vergleich zu den kontrollierten klinischen Bedingungen im OP deutlich schwieriger durchzuführen, muss sich mit limitierten Techniken begnügen und wird von medizinischen Teams durchgeführt, die häufig keine ausgewiesene Expertise in diesem Bereich besitzen.
So wurde die Einführung von extraglottischen Atemwegshilfen (EGA) auch zur primären Sicherung der Atemwege regelrecht gefeiert, da das Erlernen dieser Technik leichter ist als die Durchführung der klassischen laryngoskopischen endotrachealen Intubation (ETI). Demzufolge wurde der Einsatz auch von der Leitlinie „Präklinisches Atemwegsmanagement" aus dem Jahre 2012 empfohlen [2]. Umso ernüchternder sind die Ergebnisse der großen japanischen und US-amerikanischen Studien, die ein schlechteres Outcome präklinisch reanimierter Patienten bei Verwendung einer EGA zeigen, sowohl gegenüber der ETI als auch gegenüber der Gesichtsmaskenbeatmung (MB).

\section{Atemwegsmanagement in der Notfallmedizin}

Die endotracheale Intubation ist ein weit verbreitetes Verfahren und gilt nach wie vor in der Notfallmedizin als der „Goldstandard“ zur Sicherung der Atemwege [2,3]. Dies gilt allerdings nur mit der Einschränkung, dass die Fertigkeiten zur Durchführung hinreichend gut beherrscht werden müssen.

Die potenziellen Vorteile der endotrachealen Intubation gegenüber der Beatmung mittels einer Gesichtsmaske oder einer extraglottischen Atemwegshilfe sind in der Übersicht (s. folgende Seite oben) dargestellt [2].

Beachtet werden muss allerdings, dass die technische Durchführung der endotrachealen Intubation unter präklinischen Notfallbedingungen schwierig ist und viele Faktoren eine erfolgreiche ETI erschweren (s. Übersicht). 


\section{ÜBERSICHT}

Vorteile der endotrachealen Intubation gegenüber Verwendung einer extraglottischen Atemwegshilfe

- Applikation höherer inspiratorischer und positiv endexspiratorischer Beatmungsdrücke möglich

- geringere Leckage im Beatmungssystem

- geringere Gefahr der Insufflation von Luft in den Magen und Induktion des Circulus vitiosus der Magenbelüftung

- effektiverer Aspirationsschutz

- tracheale und bronchiale Absaugung möglich

- höhere Effektivität der Herzdruckmassage durch asynchrone Beatmung während der kardiopulmonalen Reanimation (CPR)

(mod. nach [2])

\section{ÜBERSICHT}

Faktoren, welche die präklinische endotracheale Intubation erschweren

Seitens des Patienten

- Blut, Sekrete oder Erbrochenes

- Schädigung der oberen Atemwege:

- traumatische Schäden

- thermische Schäden

- Entzündung der oberen Atemwege

- Schwellung der oberen Atemwege

- subkutane Emphyseme

- Immobilisierung der Halswirbelsäule

- Präoxygenierung eingeschränkt

- Narkosetiefe unzureichend

- keine neuromuskuläre Blockade

- Umstieg auf wache Intubationsverfahren bei der erwartet schwierigen Intubation nicht möglich

\section{Seitens des Einsatzes}

- dringlich simultan durchzuführende Tätigkeiten (z. B. kardiopulmonale Reanimation)

- ungünstige Umgebungsbedingungen (z. B. ungenügende Platzund Lichtverhältnisse, Lärm)

- Ausstattung des Rettungsmittels limitiert

- Kenntnisse und Fertigkeiten zur Sicherung der Atemwege zu gering

- keine feste Zusammensetzung der medizinischen Teams

- fehlende oder nicht an alle Beteiligten kommunizierte Standards

- kompetente Unterstützung vor Ort zeitnah nicht möglich

(mod. nach [2])
Selbst in der Intubation erfahrene Anästhesisten und Notfallmediziner stufen die präklinische ETI in 10-15\% aller Fälle als schwierig ein. Die korrespondierenden eingeschränkten Laryngoskopiegrade nach Cormack und Lehane III und IV werden mit bis zu 20\% angegeben [4]. Daraus resultierende multiple Intubationsversuche gehen mit einem erhöhten Risiko unerwünschter Ereignisse einher: Mort [5] berichtet über einen Anstieg des relativen Risikos bei Traumapatienten, die mehr als 2 Intubationsversuche im Schockraum zur endotrachealen Intubation benötigten, von

- Hypoxie (7-fach),

- schwerer Hypoxie (14-fach),

- Regurgitation (7-fach),

- Aspiration (4-fach),

- Bradykardie (4-fach),

- Herz-Kreislauf-Stillstand (7-fach).

Die Anzahl der endotrachealen Intubationen, die zum Erlernen der Technik benötigt wird, liegt zwischen 60-150 supervidierten Anwendungen [6,7]. Diese Daten beziehen sich jedoch auf die idealen Bedingungen im Operationssaal, bei Patienten ohne Risikofaktoren für die erschwerte Laryngoskopie, unter optimalen räumlichen Bedingungen und suffizienter Narkose. Nur mit Ausbildung und Supervision im notfallmedizinischen Bereich wird bereits diese geringe Anzahl an notwendigen Intubationen in der Regel nicht erreicht $[8,9]$. Die tatsächliche Anzahl der durchzuführenden ETI, die notwendig ist, um unter den erschwerten präklinischen Notfallbedingungen erfolgreich zu sein, ist bislang nicht bekannt. Es ist anzunehmen, dass diese Zahl deutlich höher liegt.

Zur sicheren Durchführung einer ETI unter Notfallbedingungen empfiehlt die „Association of Anaesthetists of Great Britain and Ireland“ eine mindestens 2-jährige Ausbildung in einem notfall- oder akutmedizinischen Fachbereich, inklusive 1 Jahr in der Anästhesiologie und Intensivmedizin [10].

Aufgrund der schwierigen Realisierung der Ausbildung ist es nicht überraschend, dass die Rate der unerkannten ösophagealen Intubationen bis zu 25\% beträgt, deren 24-Stunden-Letalität von ca. $10 \%$ bei korrekt Intubierten auf $70-90 \%$ bei Fehlintubierten steigt [2], sofern die Patienten lebend die Klinik erreichen.

\footnotetext{
Merke

Als bestmöglichen Kompromiss zwischen klinischem Anspruch und Ausbildungsrealität empfiehlt die Leitlinie der DGAI daher, dass die endotracheale Intubation nur bei $\geq 100$ angeleiteten Anwendungen und nachfolgend bei mindestens $10 \mathrm{ETI}$ pro Jahr und obligater Verwendung der Kapnografie durchgeführt werden sollte.
} 
Kann diese Anzahl nicht erfüllt werden, so wird die Anwendung von extraglottischen Atemwegen auch zur primären Atemwegssicherung empfohlen [2].

\section{Grundlagen extraglottischer Atemwege}

Die Entwicklung der Larynxmaske (LMA) als supraglottische Atemwegshilfe (SGA) wird als einer der Meilensteine der modernen Atemwegssicherung bezeichnet [11]. Seit der 1. Publikation Anfang der 1980er-Jahre wurde die klassische Larynxmaske stets weiterentwickelt.

\section{Definitionen}

Die Begrifflichkeiten „Larynxmaske“, „supra-“ und „extraglottische Atemwegshilfen“ werden in der Literatur uneinheitlich verwendet. Zu Veranschaulichung der Begrifflichkeiten sind die unterschiedlichen Methoden der Atemwegssicherung in Form von „Ebenen“ in > Abb. 1 dargestellt [12]. Der Begriff extraglottische Atemwegshilfen schließt somit die Atemwegshilfen, die außerhalb der Glottis liegen, ein. Diese werden unterschieden in die im Folgenden beschriebenen Formen.

\section{Oropharyngeale Atemwegshilfen}

Die Ebene der Atemwegshilfe befindet sich oberhalb der Glottis. Hierzu zählen die Varianten des Guedel-Tubus.

\section{Supraglottische Atemwegshilfen}

Diese Atemwegshilfe umschließt den Eingang zur Glottis. Hierzu zählen Atemwegshilfen mit aufblasbarem Cuff vom Larynxmaskentyp (LMA), z. B. LMA Classic ${ }^{\mathrm{TM}}$ (cLMA, Fa. Teleflex Medical GmbH, Kernen) oder mit festem Cuff, z. B. i-gel ${ }^{\mathrm{TM}}$ (Fa. Intersurgical, Sankt Augustin). Ebenfalls zählen hierzu die supraglottischen Atemwegshilfen der sogenannten 2. Generation, die im Weiteren näher beschrieben werden.

Der Begriff „supraglottisch“ ist aus anatomischer Sicht in diesem Zusammenhang streng genommen falsch angewendet, da bei korrekter Lage die Spitze der meisten supraglottischen Atemwegshilfen in der Postkrikoidregion platziert wird, also unterhalb der Glottisebene ( $\boldsymbol{A}$ Abb. 2). Da aber der Begriff „supraglottische Atemwegshilfen“ für diese Typen der Atemwegshilfen etabliert ist, wird er auch in diesem Artikeltext verwendet.

\section{Ebenen der Atemwegssicherung}

\section{Invasivität}

\section{A Gesichtsmaske}

B laryngopharyngeale Atemwegshilfen

C supraglottische Atemwegshilfen

B+D ösophageale Verschlusstuben extraglottische Atemwege

E Endotrachealtubus

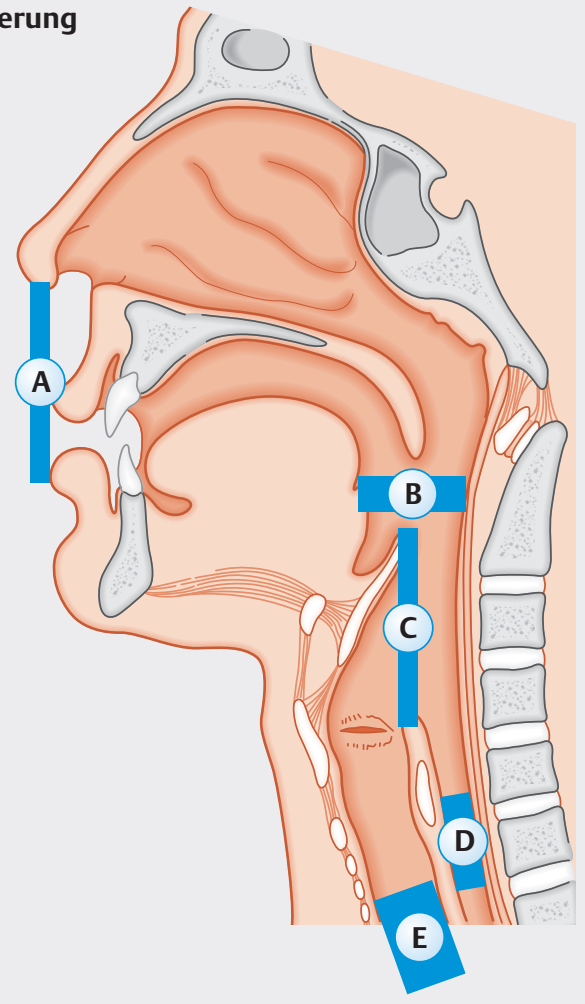

- Abb. 1 Ebenen der Atemwegsfreihaltung in Bezug auf ihre anatomische Position und Invasivität der Methoden (nach [43]). 


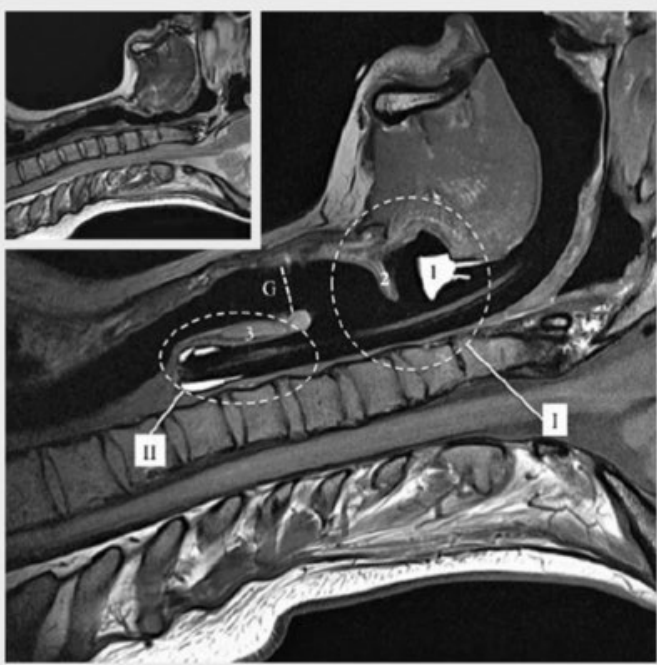

- Abb. 2 Korrekte Platzierung einer Larynxmaske LMA Supreme ${ }^{\top M}$. Der Cuff ist partiell mit Wasser gefüllt. Kleines Bild: gleicher Proband ohne Atemwegsinstrumentierung. Abkürzungen: I = Gebiet, das den oropharyngealen Leckagedruck beeinflusst (oropharyngealer Leckagedruck, OLP), II = Gebiet, das den hypopharyngealen Leckagedruck beeinflusst (hypopharyngealer Verschlussdruck [HPL], ,2nd Seal“). 1 = proximaler Cuff, 2 = Epiglottis, 3 = dorsale Seite des Krikoids, G = Ebene der Glottis. Quelle: Russo SG, Wulf H. Advanced indications of the laryngeal mask - Limitations of use. Anasthesiol Intensivmed Notfallmed Schmerzther 2014; 49: 152-161.

\section{Ösophageale Verschlusstuben}

Diese Atemwegshilfen zeichnen sich durch 2 aufblasbare Cuffs aus, wobei sich der obere Cuff analog zu den laryngopharyngealen Atemwegshilfen oberhalb der Epiglottis befindet und der untere Cuff unterhalb des Pharynx im oberen Anteil des Ösophagus, z. B. der Larynxtubus ${ }^{\mathrm{TM}}$ (LT, Fa. VBM Medizintechnik GmbH, Sulz a.N.). Die Ventilationsöffnung befindet sich zwischen beiden Cuffs.

\section{Merke}

Es sei an dieser Stelle darauf hingewiesen, dass in vielen Publikationen der Begriff „supraglottische Atemwegshilfen“ (SGA) synonym für alle extraglottischen Atemwegshilfen verwendet wird. Die vorliegende Publikation bleibt bei der korrekten Nomenklatur.

\section{Extraglottische Atemwegshilfen mit erweiterten Funktionen}

Die oben beschriebenen extraglottischen Atemwegshilfen werden stetig weiterentwickelt, vor allem, um einen besseren Aspirationsschutz und eine verbesserte Dichtigkeit zu erzielen. Hierzu zählen die supraglottischen Atemwegshilfen der 2. Generation, die nach der Definition von Tim Cook als diejenigen supraglottischen Atemwegshilfen bezeichnet wurden, die für einen verbesserten Aspi- rationsschutz konzipiert wurden [13]. Dies wird in der Regel durch einen integrierten gastrischen Kanal erreicht, über den zum einen der Ablauf regurgitierter Flüssigkeiten ermöglicht werden soll und zum anderen die Einführung einer Magensonde erleichtert wird.

\section{Merke}

Über die Magensonde kann eine aktive Entlastung von Mageninhalt (Luft, Flüssigkeiten) erfolgen.

Außerdem muss die Spitze der SGA tief in die Postkrikoidregion inserieren, um eine Separation vom Atemweg und Gastrointestinaltrakt zu erreichen ( $\bullet$ Abb. 2). So wird der Beatmungsdruck nur auf die Lunge anstatt auf den oberen Ösophagussphinkter appliziert. Diese Dichtigkeit wird auch als hypopharyngealer Verschlussdruck (HPL) bezeichnet $[14,15]$.

\section{PRINZIP}

\section{Hypopharyngealer Verschlussdruck}

Der hypopharyngeale Verschlussdruck (HPL) ist maßgeblich dafür verantwortlich, dass es bei der positiven Druckbeatmung nicht zur Insufflation von Luft in den Magen kommt und somit der Circulus vitiosus der Magenüberblähung verhindert wird [16].

Weiterhin wird durch die veränderte Passform des Cuffs in der Regel eine bessere Dichtigkeit in Richtung der Mundöffnung erzielt (OLP). Dies ermöglicht somit auch eine positive Druckbeatmung von häufig über $20 \mathrm{~cm}$ $\mathrm{H}_{2} \mathrm{O}$. Zu beachten ist hierbei, dass ein hoher oropharyngealer Leckagedruck kein Indikator für einen sicheren Sitz der supraglottischen Atemwegshilfe ist. Übersteigt der unter einem hohen oropharyngealen Leckagedruck mögliche Beatmungsdruck den HPL, so kommt es mit jeder Beatmung zwangsläufig zur Mageninsufflation mit den in $\mathbf{A}$ Abb. $\mathbf{3}$ dargestellten Folgen.

Unter Reanimationsbedingungen kommt erschwerend hinzu, dass der ösophageale Verschlussdruck rapide abnimmt. Während er beim gesunden Menschen ca. $20 \mathrm{~cm}$ $\mathrm{H}_{2} \mathrm{O}$ beträgt [17], fällt er beim Herz-Kreislauf-Stillstand schnell auf unter $5 \mathrm{~cm} \mathrm{H} \mathrm{H}_{2} \mathrm{O}$ ab [18]. Kommt es bei unerkannter Fehllage der EGA durch die Magenüberblähung zur Regurgitation, so führt dies potenziell zur Aspiration, da die extraglottische Atemwegshilfe den Abfluss nach oral versperrt.

Merke

Aus diesen Gründen muss gerade in der Notfallmedizin die korrekte Lage einer extraglottischen Atemwegshilfe überprüft werden. 


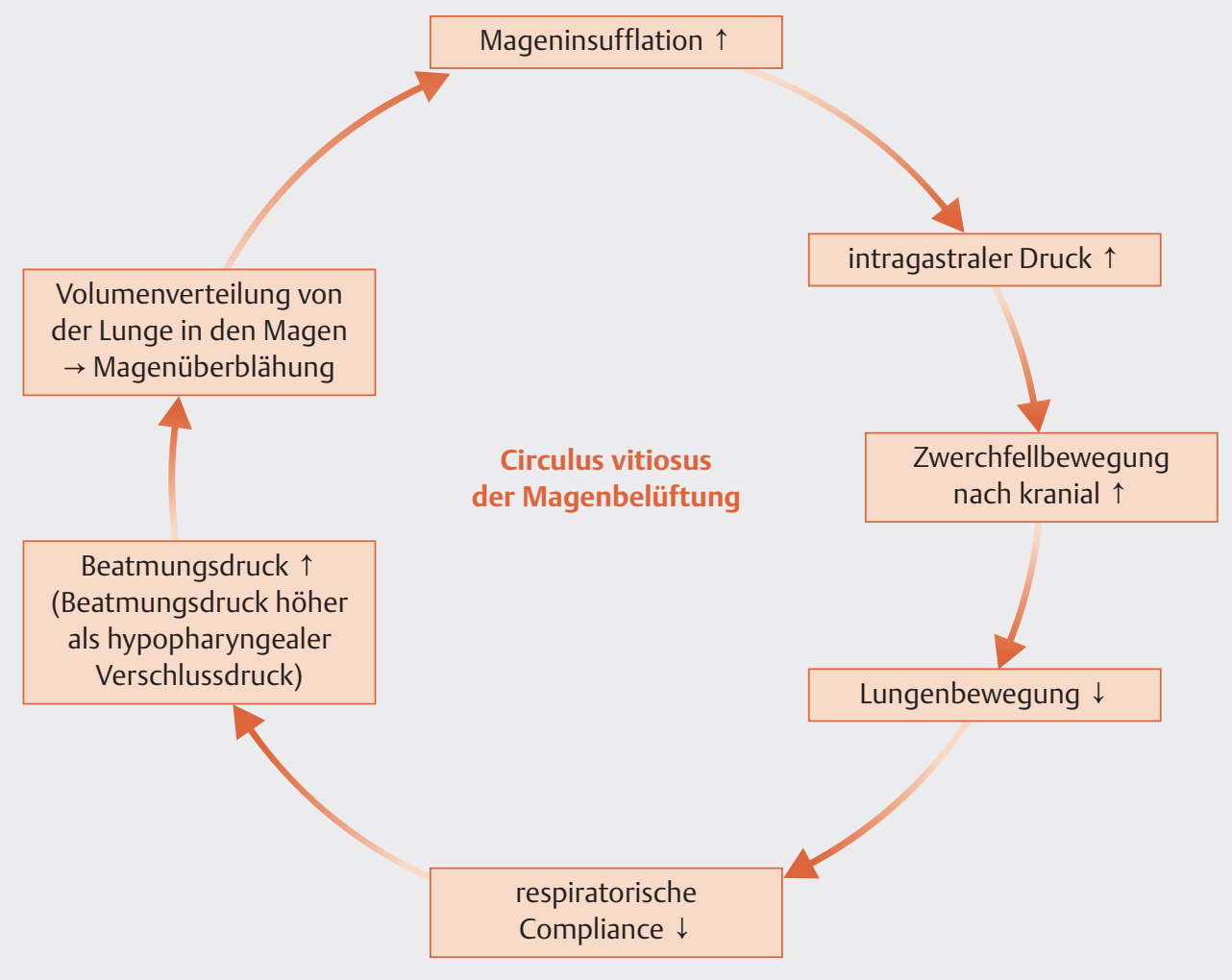

Abb. 3 Circulus vitiosus der Mageninsufflation.

Für die LMA ProSeal ${ }^{\mathrm{TM}}$ und die LMA Supreme ${ }^{\mathrm{TM}}$ sind Lagetests beschrieben worden, die Rückmeldung über eine korrekte oder falsche Platzierung geben (s. Übersicht; nach [11]). Ob diese Lagetests auch zwangsläufig für andere SGA der 2. Generation gelten, darf kritisch hinterfragt werden, da beispielsweise die i-gel ${ }^{\mathrm{TM}}$ bauartbedingt eine abgeflachte Spitze besitzt, die nicht weit in die Postkrikoidregion hineinreicht [19].

\section{PRAXIS}

Test zur Bestimmung der Lage von supraglottischen Atemwegshilfen der 2. Generation

- Beißblock mindestens zur Hälfte im Mund des Patienten (für LMA ProSeal' ${ }^{\mathrm{TM}}$ )

- negativer Magenleckagetest („Bubble-Test“)

- positiver Jugulum-Test (Supra Sternal Notch Test)

- widerstandsfreie Einlage einer Magensonde

(nach [11])
Schmidbauer und Mitarbeiter haben einen deutlich geringeren hypopharyngealen Verschlussdruck für regurgitierte Flüssigkeiten bei der i-gel ${ }^{\mathrm{TM}}\left(13 \mathrm{~cm} \mathrm{H}_{2} \mathrm{O}\right)$ im Vergleich zur LMA Classic $^{\mathrm{TM}}\left(37 \mathrm{~cm} \mathrm{H}_{2} \mathrm{O}\right)$ oder LMA ProSeal ${ }^{\mathrm{TM}}(58 \mathrm{~cm}$ $\mathrm{H}_{2} \mathrm{O}$ ) ermittelt [20]. Ob die Lagetests für den Larynxtubus mit Drainagekanal (LTS'M, Fa. VBM) verwendbar sind, ist prinzipiell denkbar, wurde aber bislang noch nicht untersucht.

\section{Potenzielle Vorteile von extraglottischen Atemwegshilfen}

Die Beatmung mit EGAs stellt in der Notfallmedizin insofern gegenüber der Gesichtsmaskenbeatmung einen Vorteil dar, als dass eine bessere Effektivität der Beatmung und größere Tidalvolumina erzielt werden können, die Mageninsufflation weniger stark ausgeprägt, die Aspirationsgefahr vermindert und die Möglichkeit der maschinellen Beatmung gegeben ist [2].

Gegenüber der endotrachealen Intubation erweist sich als Vorteil, dass die Prädiktoren der schwierigen Maskenbeatmung oder der schwierigen Laryngoskopie häufig keine Prädiktoren der schwierigen Einlage einer extraglottischen Atemwegshilfe darstellen. 
Merke

Extraglottische Atemwegshilfen stellen somit eine wertvolle Alternative bei schwieriger Maskenbeatmung oder Laryngoskopie auch für den erfahrenen Anwender dar. Der Einsatz von supraglottischen Atemwegshilfen bei schwieriger Ventilation und Intubation ist daher fester Bestandteil der internationalen Leitlinien.

Des Weiteren geben Simulationsstudien zur Reanimation Hinweise darauf, dass die Zeit der Unterbrechung der Herzdruckmassage, die für die Sicherung der Atemwege unter Verwendung einer extraglottischen Atemwegshilfe benötigt wird, niedriger ist als bei der endotrachealen Intubation, wenn diese von Ungeübten durchgeführt wird $[2,21]$.

Merke

Für die Notfallmedizin liegt der wichtigste Vorteil dieses Verfahrens jedoch in der schnelleren Erlangung basaler Fähigkeiten zur initialen Beatmung mittels eines extraglottischen Atemwegs im Vergleich zur endotrachealen Intubation.

Für die LMA Classic ${ }^{\mathrm{TM}}$ wurde bereits nach nur $10 \mathrm{kli}$ nischen Anwendungen am Patienten eine erfolgreiche Beatmungswahrscheinlichkeit von $>80 \%$ ermittelt [6] Zudem ist das Behalten der erlernten Technik nachhaltiger [22].

Die in der Notfallmedizin immer seltener zum Einsatz kommende Intubations-LMA ${ }^{\circledR}$ (Teleflex Medical GmbH, Kernen) ermöglicht unerfahrenen Anwendern eine im Vergleich zur Maskenbeatmung erfolgreichere und schnellere Etablierung der Beatmung. Ebenfalls konnten im Vergleich mit der konventionellen laryngoskopischen endotrachealen Intubation eine höhere Erfolgsrate bei der Intubation sowie eine schnellere Intubation ohne Verwendung optischer Hilfsmittel gezeigt werden [23].

PRINZIP

Lernkurve

Wichtig im Rahmen der Ausbildung ist, dass das alleinige Üben am Phantom nicht ausreicht. Die Anatomie der fragilen pharyngolaryngealen Strukturen, das Auftreten möglicher Verletzungen und die individuellen Unterschiede der Patienten und deren physiologische Antwort auf die Atemwegsmanipulation können am Manikin oder an Simulatoren nicht ausreichend genug dargestellt werden [2].

\section{Wissenschaftliche Studien}

Nachdem national und international der Einsatz von extraglottischen Atemwegshilfen aus den erwähnten Gründen stark propagiert wurde und die Handlungsabläufe auf deren Einsatz abgestimmt wurden, konnte man auf die ersten großen vergleichenden Studien gespannt sein. Hasegawa et al. untersuchten die Langzeitergebnisse der von Rettungsdienstpersonal reanimierten Patienten anhand der in Japan verpflichtenden elektronischen Dokumentation [24]. Von den 649359 eingeschlossenen Patienten wurden 57\% mittels Maskenbeatmung (MB), $37 \%$ mittels extraglottischer Atemwegshilfe und 6\% mittels endotrachealer Intubation versorgt. Nach Zuordnung der Patienten hinsichtlich ihrer Vergleichbarkeit hatten die Patienten, die nur mit einer MB versorgt wurden, die signifikant beste initiale Überlebensrate und das beste neurologische Langzeitergebnis im Vergleich zum Einsatz von extraglottischen Atemwegshilfen oder endotrachealen Tuben (ETT).

In einer von Benoit et al. durchgeführten Metaanalyse von insgesamt 10 Studien präklinisch reanimierter Patienten, die vom Rettungsdienstpersonal versorgt wurden, erhielten 34533 Patienten einen Endotrachealtubus und 41116 eine extraglottische Atemwegshilfe [25]. Auch in dieser Studie hatten Patienten, die endotracheal intubiert wurden, ein statistisch signifikant besseres Chancenverhältnis (Odds) für das Wiederauftreten eines Spontankreislaufs von 1,28, das Überleben bei Krankenhausaufnahme von 1,34 und ein neurologisch gutes Langzeitergebnis von 1,33 gegenüber den Patienten, die mit einer extraglottischen Atemwegshilfe versorgt wurden.

Beide Studien müssen allerdings dahingehend kritisch bewertet werden, dass sie nur retrospektive Auswertungen sind und auch die Art der verwendeten extraglottischen Atemwegshilfe nicht differenziert wurde. So werden mit Spannung die Ergebnisse zweier derzeit laufender prospektiv randomisierter Studien erwartet:

- die englische AIRWAYS-2-Studie, in der >9000 Patienten eingeschlossen werden sollen und die ETI mit der $\mathrm{i}$-gel ${ }^{\mathrm{TM}}$ vergleicht, und

- die US-amerikanische „PART“, in der die ETI mit dem Larynxtubus verglichen wird [26].

\section{Limitationen des Einsatzes von extraglottischen Atemwegshilfen}

Zur Analyse möglicher Schwachstellen und Limitationen des Einsatzes von extraglottischen Atemwegshilfen und endotrachealer Intubation in der Notfallmedizin können 3 Hauptkategorien identifiziert werden [27]: 


\section{Technische Fehler}

Die Durchführung einer ETI und - wenn auch in geringem Maße - die Einlage einer EGA erfordern gerade in der Notfallmedizin hochkomplexe Fertigkeiten, in welcher die technische Durchführung der Maßnahme nur einen Teilaspekt darstellt. Häufig ist eine Korrektur und Überprüfung der Handlungen erforderlich, wie beispielsweise eine Lage- und Ventilationskontrolle, die Cuffdruckmessung und ggf. erneute Durchführung des Verfahrens unter Anpassung der Einlagetechnik, der Auswahl des Atemwegs, der Lagerung oder der Notfallnarkose. Dies kann zu einer Unterbrechung der Herz-Thorax-Massage führen, die nach einer Untersuchung von Wang et al. im Mittel mehr als 11/2 min im Rahmen der ETI betrug [28].

Cheskes et al. berichten, dass bereits kurze Pausen von 20 Sekunden zu einem schlechteren Überleben führen [29]. Auch wenn Simulationsstudien eine kürzere Pause der Herzdruckmassage für die Einlage eines EGA-Systems am Manikin gegenüber der endotrachealen Intubation zeigten, so muss dies in der Realität bestätigt werden, gerade dann, wenn die oben genannten Lagetests Fehllagen aufdecken, die eine Korrektur erforderlich machen.

\section{Anatomisch-physiologische Effekte}

Der richtige Zeitpunkt der Atemwegsintervention scheint eine erwähnenswerte Rolle zu spielen. Für die Reanimation im Rahmen des Herz-Kreislauf-Stillstands postulieren Weisfeldt et al. 3 Phasen, die jeweils andere Behandlungsschwerpunkte erforderlich machen [30].

- Während der 1. elektrischen Phase steht die ggf. notwendige, sofortige Defibrillation im Vordergrund.

- In der anschließenden 2. zirkulatorischen Phase stehen die Versorgung mit Sauerstoff und ein suffizientes Atemwegsmanagement im Vordergrund.

- In der letzten metabolischen Phase ist die Verminderung des Reperfusionsschadens primäres Behandlungsziel (Hypothermie, Normoventilation).

Es muss somit darauf geachtet werden, dass die erweiterten Maßnahmen der Atemwegssicherung nicht die Defibrillation gerade der elektrischen Phasen verzögern.

Einen weiteren interessanten Aspekt bringen Segal und Mitarbeiter in die Diskussion ein. Sie konnten zeigen, dass die Einlage einer extraglottischen Atemwegshilfe (LT, LMA, Combitube) jeweils zu einer starken Abnahme des zerebralen Blutflusses unter den Niedrigflussbedingungen der Herzdruckmassage im Tiermodell führte, das bei der MB und ETI nicht beobachtet wurde [31]. Angiologische Aufnahmen zeigten als Ursache eine Kompression der Karotiden durch den Cuff der extraglottischen Atemwegshilfe. Obwohl diese Studie aus vielen methodischen Gründen kritisch zu bewerten ist, vor allem, weil das Design der EGA auf die menschliche und nicht auf die Anatomie der Versuchstiere abgestimmt ist und die Messungen des zerebralen Blutflusses unter laufender Herz-
Druck-Massage durchgeführt wurden, so weist dies doch darauf hin, dass es über die Beatmung hinaus noch weitere, bisher ungeklärte Aspekte zur Verwendung von EGAs in der Notfallsituation und vor allem während der Reanimation gibt.

Obwohl beim Menschen für die LMA Supreme ${ }^{\mathrm{TM}}$ - nicht aber für die i-gel ${ }^{\mathrm{TM}}$ - gezeigt wurde, dass die Position der Karotiden beeinflusst werden kann, so konnte eine Kompression der muskelstarken Karotiden bislang nicht nachgewiesen werden. Gleichzeitig konnten Rasulo und Kollegen keinen Einfluss auf die zerebrale Perfusion durch die Insertion einer EGA nachweisen [32].

\section{Kognitive Defizite und Ausbildungsdefizite}

Die Komplexität der Maßnahmen des erweiterten Atemwegsmanagements können auch zu einer Überkompensation anderer Maßnahmen führen, wie beispielsweise die zwingend zu vermeidende Hyperventilation oder $\mathrm{Hy}$ peroxie [33-35]. Auch die Fixierung auf die Intubation kann zu weiteren Störungen des Handlungsablaufs der kardiopulmonalen Reanimation führen. Dies wird umso ausgeprägter, je höher das Ausbildungsdefizit des Anwenders ist.

\begin{abstract}
Merke
Obwohl die Leitlinien nur 10 klinisch supervidierte Anwendungen einer extraglottischen Atemwegshilfe fordern, wird selbst diese Zahl häufig nicht erreicht. Ursächlich sind häufig die mangelnde Abstimmung der innerklinischen Ausbildungsmöglichkeiten und die Ausrüstung der Rettungsmittel.
\end{abstract}

Aus einer eigenen Umfrage an Ausbildungskrankenhäusern und NEF-/RTW-Standorten in Berlin und Brandenburg geht hervor, dass das innerklinische Training zu fast $80 \%$ mit supraglottischen Atemwegshilfen vom klassischen Larynxmaskentyp durchgeführt wird und nur in gut $10 \%$ mit dem Larynxtubus. Hinsichtlich der Ausstattung der Rettungsmittel verhält es sich genau umgekehrt: In ca. $80 \%$ wird der Larynxtubus vorgehalten, während supraglottische Atemwegshilfen nur in ca. $20 \%$ vorhanden sind. Hier ist eine dringende Angleichung zwischen den Ausbildungsstätten und den Rettungsdiensten erforderlich.

PRINZIP

\section{Ausrüstung im Rettungsdienst}

Die Ausrüstung der Rettungsmittel hinsichtlich der Art der extraglottischen Atemwegshilfe muss mit den Ausbildungsmöglichkeiten der Krankenhäuser abgestimmt werden. 
Bernhard und Mitarbeiter beschreiben in einer Metaanalyse weiterhin, dass die Insertion einer supraglottischen Atemwegshilfe vom Larynxmaskentyp erfolgreicher ist als die des Larynxtubus [36]. In den 26 eingeschlossenen Studien mit 2159 Patienten wurde die erfolgreiche Intubation im 1. Versuch und insgesamt für den Larynxtubus mit 77 und $92 \%$ vs. 79 und $98 \%$ mit einer Larynxmaske angeben. Die zugrunde liegenden Studien haben das Ergebnis „erfolgreich“ für beide Typen extraglottischer Atemwegshilfen allerdings lediglich auf die initiale Beatmungsmöglichkeit bezogen. Eine Lagekontrolle, die besonders für den längeren Einsatz bei potenziell nicht nüchternen Patienten unbedingt zu fordern ist, erfolgte nicht.

\section{Spezifische Komplikationen von extraglottischen Atemwegshilfen}

Auch müssen die Effekte möglicher Fehllagen und traumatischer Verletzungen auf das Langzeitergebnis diskutiert werden. Gerade die unerkannten Fehlpositionen einer extraglottischen Atemwegshilfe können neben einer schweren Hypoventilation vor allem zu einer massiven gastrischen Überblähung führen. Mann et al. [37] beschreiben in einem Fallbericht die Folge: Die Insufflation von insgesamt 3 I Luft in den Magen führte bei einem verunfallten und mit einem Larynxtubus versorgten Patienten zu einer deutlichen Hypoventilation.

In einer Fallserie von 189 mit einem Larynxtubus versorgten Patienten wurde in $20 \%$ der Fälle eine deutliche Magenüberblähung festgestellt [38]. Die weiteren Folgen können eine Magenruptur oder eine Kreislaufdepression infolge des verminderten Rückstroms aus der komprimierten V. cava inferior bis zum Kreislaufstillstand sein.

\section{PRINZIPIEN}

\section{Bedingungen für den Einsatz von SGA}

- In der Notfallmedizin sollten ausschließlich supraglottische Atemwegshilfen der 2. Generation verwendet werden.

- Neben den Tests zur Lagekontrolle sollte der Magen obligat durch eine Magensonde entlastet werden.

Eine weitere speziell bei den extraglottischen Atemwegshilfen vom Typ Larynxtubus berichtete Komplikation ist die Zungenschwellung [39]. Diese wurde in 39\% aller präklinischen Anwendungen beobachtet und kann zu schwerwiegenden Komplikationen bei der Umintubation führen, welche den Einsatz der Videolaryngoskopie, eines starren Endoskops bis hin zur Koniotomie erforderlich machten [38].
Cave

Bei der Umintubation von einem Larynxtubus auf einen Endotrachealtubus können Intubationsschwierigkeiten auftreten.

Im Rahmen des innerklinischen Atemwegsmanagements könnte somit mit weiteren iatrogen induzierten hypoxämischen Phasen bis zum sekundären Herz-Kreislauf-Stillstand gerechnet werden, mit ungünstiger Auswirkung auf das Langzeitergebnis. Weitere Untersuchungen müssen zeigen, ob die von Schalk et al. empfohlene Cuffdruckkontrolle die Häufigkeit dieser Komplikation reduziert [38].

\begin{abstract}
Merke
Nach Einlage einer extraglottischen Atemwegshilfe

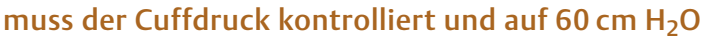
begrenzt werden.
\end{abstract}

\section{Einsatz von extraglottischen Atemwegshilfen bei Kindern}

Besonders erwähnenswert ist die Empfehlung zum Einsatz des Larynxtubus ${ }^{\mathrm{TM}}$ (LT, Fa. VBM Medizintechnik $\mathrm{GmbH}$, Sulz a. N.) bei Kindern, die unter anderem vom wissenschaftlichen Arbeitskreis Kinderanästhesie der DGAI publiziert wurde [40]. Hier wird der Einsatz des Larynxtubus weder in der Routine- noch in der Notfallsituation - besonders für Kinder unter $10 \mathrm{kgKG}$ - empfohlen.

Sie führen in ihrer Begründung an, dass eine korrekte Platzierung des Larynxtubus aufgrund des bei Kindern hoch sitzenden Kehlkopfs sowie der proportional größeren Zunge schwieriger erreichbar ist als bei Erwachsenen. Da zudem im klinischen Alltag der LT sehr selten zum Einsatz kommt, ist auch bei größeren Kindern kein regelmäßiges Training sowohl für ärztliches als auch für nicht ärztliches Personal gegeben.

\section{Anwendungsprobleme des Larynxtubus}

- Das Konzept des Doppelcuffs scheint nicht nur bei Kindern mit Anwendungsproblemen behaftet zu sein.

- Bei unzureichender Insertionstiefe besteht die Gefahr, dass der distale Cuff zu einer Atemwegsobstruktion führt und zudem eine Luftinsufflation in den Gastrointestinaltrakt stattfindet.

- Bei zu tiefer Insertionstiefe hingegen kann es durch den proximalen Cuff zu einer Verlegung der Glottis kommen.

- Zusätzlich kann es durch Verdrehungen des Larynxtubus in seiner Längsachse zu einer Obstruktion der Ventilationsöffnungen mit konsekutiver Verschlechterung der Ventilationsbedingungen kommen. 
- Schließlich kann das blind endende Lumen des LT auch akzidentell in die Trachea eingeführt werden und damit den Atemweg komplett verschließen [41,42].

Die Empfehlung der DGAI schließt mit der folgenden Stellungnahme:

\section{PRAXIS}

\section{Empfehlung der DGAI}

Unter Berücksichtigung der wissenschaftlichen Datenlage und der großen, langjährigen klinischen Erfahrung mit der Larynxmaske in der Elektiv- und Notfallanwendung bei Kindern kann derzeit zum alternativen Atemwegsmanagement im Kindesalter mit einer supraglottischen Atemwegshilfe (SGA) ausschließlich die Larynxmaske empfohlen werden.

\section{Bewertung der aktuellen Situation}

Die anfänglichen Hoffnungen, dass die ETI durch den Einsatz von EGAs in der Notfallmedizin ersetzt werden kann, werden derzeit durch die aktuelle Studienlage bei präklinisch reanimierten Patienten nicht belegt. Obwohl potenzielle Vorteile durch das schnellere Erlernen der Technik vermutet werden, spiegelt sich dies in Bezug auf das Langzeitergebnis nicht wider.

Problematisch erscheint auch die mangelnde Ausbildung am Patienten, gepaart mit einer fehlenden Abstimmung zwischen den Ausbildungsstätten und den Ausrüstungen der Rettungsmittel. Welchen Einfluss die Wahl der verwendeten EGAs auf das Langzeitergebnis von Patienten hat, ist derzeit unklar. Aufgrund aktueller Empfehlungen und Publikationen darf die derzeitige Dominanz des Larynxtubus (LT) als präferierte extraglottische Atemwegshilfe in der Notfallmedizin kritisch hinterfragt werden.

Für andere SGA bestehen diverse Vorteile:

- Es besteht deutlich mehr klinische Erfahrung.

- Die Vorhaltung und innerklinische Ausbildung am Patienten von verschiedenen EGA-Typen ist nicht notwendig.

- Der Einsatz wird auch bei Kindern empfohlen.

- Sie erscheinen leichter zu inserieren.

- Sie sind potenziell mit weniger Komplikationen behaftet.

\section{KERNAUSSAGEN}

- Der Goldstandard der Atemwegssicherung ist weiterhin die endotracheale Intubation.

- Extraglottische Atemwegshilfen (EGA) haben vor allem Vorteile im schnelleren Erlernen der Platzierung im Vergleich zur laryngoskopischen endotrachealen Intubation.

- Wenn extraglottische Atemwegshilfen verwendet werden, dann sollten

- zwingend die der 2. Generation verwendet werden,

- der Cuffdruck auf $60 \mathrm{~cm} \mathrm{H} \mathrm{H}_{2} \mathrm{O}$ begrenzt werden,

- die Tests zur Lagebestimmung obligat durchgeführt werden.

- Wissenschaftliche Studien zeigen bislang bei präklinisch reanimierten Patienten ein schlechteres Langzeitergebnis bei Verwendung einer extraglottischen Atemwegshilfe im Vergleich zur Maskenbeatmung und endotrachealer Intubation.

- Das schlechtere Abschneiden der extraglottischen Atemwegshilfe könnte an der unzureichenden Ausbildung, der fehlenden Lagekontrolle, der fehlenden Einlage einer Magensonde und an für den jeweiligen EGA spezifischen Komplikationen liegen.

- Die innerklinische Ausbildung muss mit der Ausrüstung der Rettungsmittel abgestimmt werden.

- Im Vergleich mit dem Larynxtubus zeichnen sich andere supraglottische Atemwegshilfen (SGA) aus durch

- eine schnellere und erfolgreichere Einlage,

- weniger Komplikationen und

- ein breiteres Anwendungsspektrum.

\section{Interessenkonflikt}

Die Autoren geben an, dass kein Interessenkonflikt besteht. 
Über die Autoren

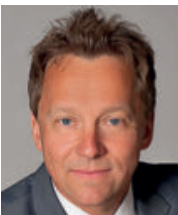

\section{Arnd Timmermann}

Prof. Dr. med. DEAA, MME, Jahrgang 1966. Bis 1995 Studium der Medizin. Facharztausbildung und Promotion am Zentrum Anästhesiologie, Rettungs- und Intensivmedizin der Universitätsmedizin Göttingen. 2007 Habilitation zum Thema „Untersuchungen zum präklinischen Atemwegsmanagement und zu der Intubationslarynxmaske als alternativer Methode der Atemwegssicherung“. 2007 Abschluss als Master of Medical Education an der Universität Heidelberg. Seit 2012 Chefarzt der Klinik für Anästhesie, Intensiv- und Notfallmedizin und Schmerztherapie, OP-Management an den DRK Kliniken Berlin Westend und Mitte. Hauptforschungsschwerpunkt: Atemwegsmanagement. Executive Director der European Airway Management Society.

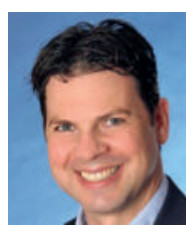

\section{Sebastian G. Russo}

Prof. Dr. med. Sebastian G. Russo, MHM, DEAA. Bis 2000 Studium der Humanmedizin an der Universitätsmedizin Göttingen. Facharztausbildung an der Klinik für Anästhesiologie, Universitätsmedizin Göttingen. 2012 Habilitation zum Thema „Evaluation und Untersuchung erweiterter Einsatzmöglichkeiten moderner Larynxmasken. 2014-2017 Geschäftsführender Oberarzt der Klinik für Anästhesiologie, Universitätsmedizin Göttingen. Seit 2017 Direktor der Klinik für Anästhesiologie am Lehrstuhl für Anästhesiologie I der Universität Witten/Herdecke. 2. Sprecher des wissenschaftlichen Arbeitskreises Atemwegsmanagement der DGAI.

\section{Korrespondenzadresse}

Prof. Dr. med. Arnd Timmermann

Klinik für Anästhesie, Intensiv- und Notfallmedizin und Schmerztherapie, OP-Management

DRK Kliniken Berlin Westend

Spandauer Damm 130

14050 Berlin

a.timmermann@drk-kliniken-berlin.de

Literatur

[1] Dirks B. Sicherung der Atemwege in der Notfallmedizin. Stuttgart: Thieme; 2001

[2] Timmermann A, Byhahn C, Wenzel V et al. Handlungsempfehlung für das präklinische Atemwegsmanagement. Für Notärzte und Rettungsdienstpersonal. Anästh Intensivmed 2012; 53: 294-308

[3] Soar J, Nolan JP, Bottiger BW et al. European Resuscitation Council Guidelines for resuscitation 2015: section 3. Adult advanced life support. Resuscitation 2015; 95: 100-147

[4] Timmermann A, Eich C, Russo SG et al. Prehospital airway management: A prospective evaluation of anaesthesia trained emergency physicians. Resuscitation 2006; 70: 179-185

[5] Mort TC. Emergency tracheal intubation: complications associated with repeated laryngoscopic attempts. Anesth Analg 2004; 99: 607-613

[6] Bernhard M, Mohr S, Weigand MA et al. Developing the skill of endotracheal intubation: implication for emergency medicine. Acta Anaesthesiol Scand 2012; 56: 164-171
[7] Komatsu R, Kasuya $\mathrm{Y}$, Yogo $\mathrm{H}$ et al. Learning curves for bagand-mask ventilation and orotracheal intubation: an application of the cumulative sum method. Anesthesiology 2010; 112: $1525-1531$

[8] Johnston BD, Seitz SR, Wang HE. Limited opportunities for paramedic student endotracheal intubation training in the operating room. Acad Emerg Med 2006; 13: 1051-1055

[9] Timmermann A, Braun U, Panzer W et al. Präklinisches Atemwegsmanagement in Norddeutschland: Individuelle Kenntnisse, Vorgehen und Ausrüstung. Anaesthesist 2007; 56: 328334

[10] Association of Anaesthetists of Great Britain and Ireland. AAGBI Safety guideline pre-hospital anaesthesia. London: The Association of Anaesthetists of Great Britain and Ireland; 2009. Im Internet: https://www.aagbi.org/sites/default/files/prehospital_glossy09.pdf; Stand: 13.04.2017

[11] Timmermann A, Nickel EA, Puhringer F. Larynxmasken der zweiten Generation: Erweiterte Indikationen. Anaesthesist 2015; 64: 7-15

[12] Timmermann A. Supraglottic airways in difficult airway management: successes, failures, use and misuse. Anaesthesia 2011; 66 (S2): 45-56

[13] Cook TM, Howes B. Supraglottic airway devices: recent advances. Contin Educ Anaesth Crit Care Pain 2011; 2: 56-61

[14] Russo SG, Wulf H. Advanced indications of the laryngeal mask - Limitations of use. Anasthesiol Intensivmed Notfallmed Schmerzther 2014; 49: 152-161

[15] Timmermann A, Bergner UA, Russo SG. Laryngeal mask airway indications: new frontiers for second-generation supraglottic airways. Curr Opin Anaesthesiol 2015; 28: 717-726

[16] Wenzel V, Idris AH, Banner MJ et al. Influence of tidal volume on the distribution of gas between the lungs and stomach in the nonintubated patient receiving positive-pressure ventilation. Crit Care Med 1998; 26: 364-368

[17] Ruben H, Knudsen E], Carugati G. Gastric inflation in relation to airway pressure. Acta Anaesthesiol Scand 1961; 5: 107-114

[18] Bowman FP, Menegazzi J], Check BD et al. Lower esophageal sphincter pressure during prolonged cardiac arrest and resuscitation. Ann Emerg Med 1995; 26: 216-219

[19] Russo SG, Cremer S, Eich C et al. Magnetic resonance imaging study of the in vivo position of the extraglottic airway devices $\mathrm{i}$-gel and LMA-Supreme in anaesthetized human volunteers. $\mathrm{Br}$ J Anaesth 2012; 109: 996-1004

[20] Schmidbauer W, Bercker S, Volk T et al. Oesophageal seal of the novel supralaryngeal airway device I-Gel in comparison with the laryngeal mask airways Classic and ProSeal using a cadaver model. Br ] Anaesth 2009; 102: 135-139

[21] Russo SG, Stradtmann C, Crozier TA et al. Bag-mask ventilation and direct laryngoscopy versus intubating laryngeal mask airway: a manikin study of hands-on times during cardiopulmonary resuscitation. Eur J Emerg Med 2014; 21: 189-194

[22] Ruetzler K, Roessler B, Potura L et al. Performance and skill retention of intubation by paramedics using seven different airway devices - a manikin study. Resuscitation 2011; 82: 593597

[23] Timmermann A, Russo SG, Crozier TA et al. Novices ventilate and intubate quicker and safer via intubating laryngeal mask than by conventional bag-mask ventilation and laryngoscopy. Anesthesiology 2007; 107: 570-576

[24] Hasegawa K, Hiraide A, Chang Y et al. Association of prehospital advanced airway management with neurologic outcome and survival in patients with out-of-hospital cardiac arrest. JAMA 2013; 309: 257-266 
[25] Benoit JL, Gerecht RB, Steuerwald MT et al. Endotracheal intubation versus supraglottic airway placement in out-of-hospital cardiac arrest: A meta-analysis. Resuscitation 2015; 93: 20-26

[26] Taylor J, Black S, Brett J et al. Design and implementation of the AIRWAYS-2 trial: A multi-centre cluster randomised controlled trial of the clinical and cost effectiveness of the i-gel supraglottic airway device versus tracheal intubation in the initial airway management of out of hospital cardiac arrest. Resuscitation 2016; 109: 25-32

[27] Benoit JL, Prince DK, Wang HE. Mechanisms linking advanced airway management and cardiac arrest outcomes. Resuscitation 2015; 93: 124-127

[28] Wang HE, Simeone SJ, Weaver MD et al. Interruptions in cardiopulmonary resuscitation from paramedic endotracheal intubation. Ann Emerg Med 2009; 54: 645-652

[29] Cheskes S, Schmicker RH, Verbeek PR et al. The impact of perishock pause on survival from out-of-hospital shockable cardiac arrest during the Resuscitation Outcomes Consortium PRIMED trial. Resuscitation 2014; 85: 336-342

[30] Weisfeldt ML, Becker LB. Resuscitation after cardiac arrest: a 3-phase time-sensitive model. JAMA 2002; 288: 3035-3038

[31] Segal N, Yannopoulos D, Mahoney BD et al. Impairment of carotid artery blood flow by supraglottic airway use in a swine model of cardiac arrest. Resuscitation 2012; 83: 1025-1030

[32] Rasulo F, Zugni N, Piva S et al. Influence of supraglottic airway device placement on cerebral hemodynamics. Minerva Anestesiol 2016; 82: 850-857

[33] Gaither JB, Spaite DW, Bobrow B] et al. Balancing the potential risks and benefits of out-of-hospital intubation in traumatic brain injury: the intubation/hyperventilation effect. Ann Emerg Med 2012; 60: 732-736

[34] Aufderheide TP, Sigurdsson G, Pirrallo RG et al. Hyperventilation-induced hypotension during cardiopulmonary resuscitation. Circulation 2004; 109: 1960-1965
[35] Kilgannon JH, Jones AE, Shapiro NI et al. Association between arterial hyperoxia following resuscitation from cardiac arrest and in-hospital mortality. JAMA 2010; 303: 2165-2171

[36] Bernhard M, Gries A, Ramshorn-Zimmer A et al. Insertion success of the laryngeal tube in emergency airway management. Biomed Res Int 2016; 2016: 3619159

[37] Mann V, Mann ST, Alejandre-Lafont E et al. Supraglottische Atemwegshilfen in der Notfallmedizin: Stellenwert der Magendrainage. Anaesthesist 2013; 62: 285-292

[38] Schalk R, Seeger FH, Mutlak H et al. Complications associated with the prehospital use of laryngeal tubes - a systematic analysis of risk factors and strategies for prevention. Resuscitation 2014; 85: 1629-1632

[39] Bernhard M, Beres W, Timmermann A et al. Prehospital airway management using the laryngeal tube. An emergency department point of view. Anaesthesist 2014; 63: 589-596

[40] Hoffmann F, Keil J, Urban B et al. Atemwegsmanagement mit supraglottischen Atemwegshilfen in der Kindernotfallmedizin. Anästh Intensivmed 2016; 57: 377-386

[41] Byhahn C, Schalk R, Russo SG. Präklinische Atemwegssicherung. Drama in 5 Akten. Anaesthesist 2014; 63: 543-545

[42] Russo SG, Cremer S, Galli T et al. Randomized comparison of the $\mathrm{i}-\mathrm{ge}^{\mathrm{TM}}$, the LMA Supreme ${ }^{\mathrm{TM}}$, and the Laryngeal Tube Suction-D using clinical and fibreoptic assessments in elective patients. BMC Anesthesiology 2012; 12: 18

[43] Timmermann A. Supraglottic airways in difficult airway management: successes, failures, use and misuse. Anaesthesia 2011; 66 (Suppl. 2): 45-56

Bibliografie

DOI https://doi.org/10.1055/s-0043-108525

Notfallmedizin up2date 2017; 12: 143-155

(c) Georg Thieme Verlag KG Stuttgart · New York

ISSN 1611-6550 
Punkte sammeln auf CME. thieme.de

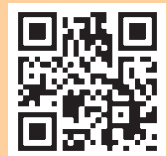

Diese Fortbildungseinheit ist 12 Monate online für die Teilnahme verfügbar.

Sollten Sie Fragen zur Online-Teilnahme haben, finden Sie unter cme.thieme.de/hilfe eine ausführliche Anleitung. Wir wünschen viel Erfolg beim Beantworten der Fragen!

Unter eref.thieme.de/ZZX8S3S oder über den QR-Code kommen Sie direkt zum Artikel zur Eingabe der Antworten.

VNR 2760512017152372407

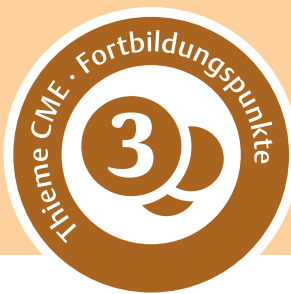

\section{Frage 1}

Wodurch ist das Outcome präklinisch reanimierter Patienten gekennzeichnet?

A Zwischen den Beatmungsformen präklinisch reanimierter Patienten konnte kein Unterschied im Outcome gefunden werden.

B Sowohl die präklinische Beatmung durch Gesichtsmaske als auch die endotracheale Intubation zeigten ein schlechteres Outcome als extraglottische Atemwegshilfen.

C Nach Maskenbeatmung resultierte ein besseres Outcome als nach Einsatz extraglottischer Atemwegshilfen.

D Extraglottische Atemwegshilfen schnitten besser ab als die endotracheale Intubation.

E Die Form der Beatmung bezogen auf das Outcome wurde noch nicht in repräsentativen Studien untersucht.

\section{Frage 2}

Welches Verfahren gilt als Goldstandard zur Sicherung der Atemwege?

A Larynxmaske

B Guedel-Tubus

$C$ endotracheale Intubation

D Videolaryngoskop

E extraglottische Atemwegshilfen

\section{Frage 3}

In welcher Liste ist die zunehmende Invasivität der Beatmungsformen dargestellt?

A laryngopharyngeale Atemwegshilfen - ösophagealer Verschlusstubus - supraglottische Atemwegshilfen

B Maskenbeatmung/Gesichtsmaske - laryngopharyngeale Atemwegshilfen - supraglottische Atemwegshilfen - Endotrachealtubus

C Maskenbeatmung/Gesichtsmaske - laryngopharyngeale Atemwegshilfen - ösophagealer Verschlusstubus - supraglottische Atemwegshilfen

D Maskenbeatmung/Gesichtsmaske - supraglottische Atemwegshilfen - laryngopharyngeale Atemwegshilfen - Endotrachealtubus

E supraglottische Atemwegshilfen - laryngopharyngeale Atemwegshilfen - ösophagealer Verschlusstubus - Endotrachealtubus

\section{Frage 4}

Wie lauten die Ausbildungsempfehlungen der Deutschen Gesellschaft für Anästhesiologie und Intensivmedizin (DGAI) hinsichtlich der Atemwegssicherung?

A Die tatsächliche Anzahl der durchzuführenden ETI, die notwendig ist, um unter den erschwerten präklinischen Notfallbedingungen erfolgreich zu sein, wurde mit 50 Eingriffen festgelegt.

B Eine ausreichende Expertise ist nach 10 endotrachealen Intubationen erreicht.

C Sofern eine kapnografische Kontrolle erfolgt, ist jeder Mitarbeiter des Rettungsdienstes berechtigt, eine endotracheale Intubation durchzuführen.

D Die eigenständige endotracheale Intubation sollte erst nach $\geq 100$ angeleiteten Anwendungen durchgeführt werden.

E Jeder rettungsdienstlich tätige Kollege führt die endotracheale Intubation routinemäßig durch.

\section{Frage 5}

Zu den Vorteilen der endotrachealen Intubation gegenüber der Verwendung einer extraglottischen Atemwegshilfe gehört einer der folgenden Aspekte nicht. Welcher?

A effektiverer Aspirationsschutz

B geringere Gefahr der Magenbelüftung

C leichtere Erlernbarkeit

D tracheale und bronchiale Absaugung möglich

E höhere Dichtigkeit im Beatmungssystem

\section{- Weitere Fragen auf der folgenden Seite...}




\section{Punkte sammeln auf CME. thieme.de}

Fortsetzung...

\section{Frage 6}

Supraglottische Atemhilfen der 2. Generation zeichnen sich dadurch aus, dass ...

A sie eine erleichterte Implementation zum primären Ziel der Entwicklung hatten.

B sie ohne gastrischen Kanal auskommen.

C sie wegen der geringeren Dichtigkeit verglichen mit dem Vorgängermodell wieder aufgegeben wurden.

D sie mit dem Ziel eines besseren Aspirationsschutzes konzipiert wurden.

E die Tubusspitze deutlich oberhalb der Postkrikoidregion zu liegen kommen muss.

\section{Frage 7}

Eine der folgenden Aussagen trifft nicht zu. Welche?

A Ist eine endotracheale Intubation geplant, sollte der spontanatmende Patient bis zu 3 Minuten präoxygeniert werden.

B Zu den absoluten Kontraindikationen für eine nicht invasive Beatmung gehören gastrointestinale Blutungen oder lleus.

C Die Anwendung eines Guedel-Tubus erfordert eine ausreichend tiefe Hypnose des Patienten für die Toleranz, sonst drohen Würgen bzw. Erbrechen.

D Die DGAI empfiehlt bei Kindern in der Elektiv- wie auch in der Notfallanwendung zum alternativen Atemwegsmanagement mit einer supraglottischen Atemwegshilfe (SGA) ausschließlich die Larynxmaske.

E Es ist präklinisch nicht möglich, den korrekten Sitz einer extraglottischen Atemwegshilfe zu überprüfen.

\section{Frage 8}

Eines der folgenden Probleme gehört nicht zu den spezifischen Komplikationen extraglottischer Atemwegshilfen. Welches?
A Schwierigkeiten bei der Umintubation
B iatrogene Verletzung bei der bronchialen Absaugung
C Hypoventilation
D Fehllage der Atemwegshilfe
E iatrogene Verletzung während der Applikation

\section{Frage 9}

Welchen Vorteil bietet der Einsatz extraglottischer Atemwegshilfen in der Notfallmedizin gegenüber der Beatmung mit einer Gesichtsmaske?

A geringere Fehlerträchtigkeit

B größere Aspirationsgefahr

C Mageninsufflation stärker ausgeprägt

D Möglichkeit, eine maschinelle Beatmung zuzuschalten

E Tidalvolumina kleiner

\section{Frage 10}

Einer der folgenden Faktoren ist nicht am sogenannten Circulus vitiosus der Mageninsufflation beteiligt. Welcher?
A Volumenumverteilung (Lunge $\rightarrow$ Magen)
$B$ ein erhöhter intragastraler Druck
C eine geringere respiratorische Compliance
D ein möglichst niedriger oropharyngealer Leckagedruck
E eingeschränkte pulmonale Bewegung 